\title{
ON FUNCTIONAL EQUATIONS RELATED TO DERIVATIONS IN SEMIPRIME RINGS AND STANDARD OPERATOR ALGEBRAS
}

\author{
NeJC ŠIrovNIK \\ University of Maribor, Slovenia
}

\begin{abstract}
In this paper functional equations related to derivations on semiprime rings and standard operator algebras are investigated. We prove, for example, the following result, which is related to a classical result of Chernoff. Let $X$ be a real or complex Banach space, let $L(X)$ be the algebra of all bounded linear operators of $X$ into itself and let $A(X) \subset L(X)$ be a standard operator algebra. Suppose there exist linear mappings $D, G: A(X) \rightarrow L(X)$ satisfying the relations $D\left(A^{3}\right)=$ $D\left(A^{2}\right) A+A^{2} G(A), G\left(A^{3}\right)=G\left(A^{2}\right) A+A^{2} D(A)$ for all $A \in A(X)$. In this case there exists $B \in L(X)$ such that $D(A)=G(A)=[A, B]$ holds for all $A \in A(X)$.
\end{abstract}

Throughout, $R$ will represent an associative ring with center $Z(R)$. As usual we write $[x, y]$ for $x y-y x$. Given an integer $n \geq 2$, a ring $R$ is said to be $n$-torsion free, if for $x \in R, n x=0$ implies $x=0$. Recall that a ring $R$ is prime, if for $a, b \in R, a R b=(0)$ implies $a=0$ or $b=0$, and is semiprime in case $a R a=(0)$ implies $a=0$. We denote by $Q_{s}$ the symmetric Martindale ring of quotients. For the explanation of $Q_{s}$ we refer the reader to [2]. Let $A$ be an algebra over the real or complex field and let $B$ be a subalgebra of $A$. A linear mapping $D: B \rightarrow A$ is called a linear derivation in case $D(x y)=D(x) y+x D(y)$ holds for all pairs $x, y \in R$. In case we have a ring $R$, an additive mapping $D: R \rightarrow R$ is called a derivation, if $D(x y)=D(x) y+x D(y)$ holds for all pairs $x, y \in R$ and is called a Jordan derivation in case $D\left(x^{2}\right)=D(x) x+x D(x)$ is fulfilled for all $x \in R$. A derivation $D$ is inner in case there exists $a \in R$ such that $D(x)=[x, a]$ holds

2010 Mathematics Subject Classification. 16N60, 46B99, 39B42.

Key words and phrases. Prime ring, semiprime ring, Banach space, standard operator algebra, derivation, Jordan derivation, Jordan triple derivation.

This research has been supported by the Research Council of Slovenia. 
for all $x \in R$. Every derivation is a Jordan derivation. The converse is in general not true. A classical result of Herstein ([9]) asserts that any Jordan derivation on a 2 -torsion free prime ring is a derivation. A brief proof of Herstein's result can be found in [5]. Cusack ([8]) generalized Herstein's result to 2 -torsion free semiprime rings (see also [3] for an alternative proof). For results in the spirit of Herstein's theorem we refer to [1,6,10,11]. An additive mapping $D: R \rightarrow R$, where $R$ is an arbitrary ring, is called a Jordan triple derivation in case $D(x y x)=D(x) y x+x D(y) x+x y D(x)$ holds for all pairs $x, y \in R$. One can easily prove that any Jordan derivation $D$ on an arbitrary 2 -torsion free ring $R$ is a Jordan triple derivation (see, for example, [5]). Let $X$ be a real or complex Banach space and let $L(X)$ and $F(X)$ denote the algebra of all bounded linear operators on $X$ and the ideal of all finite rank operators in $L(X)$, respectively. An algebra $A(X) \subset L(X)$ is said to be standard in case $F(X) \subset A(X)$. Let us point out that any standard operator algebra is prime, which is a consequence of Hahn-Banach theorem.

Brešar ([4]) has proved the following result.

TheOREM 1. Let $R$ be a 2 -torsion free semiprime ring and let $D: R \rightarrow R$ be a Jordan triple derivation. In this case $D$ is a derivation.

Motivated by Theorem 1, Vukman, Kosi-Ulbl and Eremita ([16]) have proved the following result.

THEOREM 2. Let $R$ be a 2-torsion free semiprime ring. Suppose there exists an additive mapping $T: R \rightarrow R$ such that

$$
T(x y x)=T(x) y x-x T(y) x+x y T(x)
$$

for all $x, y \in R$. Then there exists $q \in Q_{s}$ such that $2 T(x)=q x+x q$ for all $x \in R$.

In the same article, Vukman, Kosi-Ulbl and Eremita proved the result below, which is an immediate consequence of Theorem 1 and Theorem 2 .

Corollary 3. Let $R$ be a 2 -torsion free semiprime ring. If $D, G: R \rightarrow$ $R$ are additive mappings such that

$$
\begin{aligned}
& D(x y x)=D(x) y x-x G(y) x+x y D(x), \\
& G(x y x)=G(x) y x-x D(y) x+x y G(x)
\end{aligned}
$$

for all $x \in R$, then there exists a derivation $S: R \rightarrow R$ and $q \in Q_{s}$ such that

$$
\begin{aligned}
& 4 D(x)=q x+x q+S(x), \\
& 4 G(x)=q x+x q-S(x)
\end{aligned}
$$

for all $x \in R$.

Theorem 1 also motivated Vukman ([20]), who has recently proved the following result. 
THEOREM 4. Let $R$ be a 2 -torsion free semiprime ring and let $D: R \rightarrow R$ be an additive mapping. Suppose that either

$$
D(x y x)=D(x y) x+x y D(x)
$$

or

$$
D(x y x)=D(x) y x+x D(y x)
$$

holds for all pairs $x, y \in R$. In both cases $D$ is a derivation.

It is our aim in this paper to generalize Theorem 4, but first we will prove the following theorem, which is conceptually linked with Theorem 2 .

TheOREM 5. Let $R$ be a 2 -torsion free semiprime ring and let $T: R \rightarrow R$ be an additive mapping. Suppose that either

$$
T(x y x)=T(x y) x-x y T(x)
$$

$$
T(x y x)=T(x) y x-x T(y x)
$$

holds for all pairs $x, y \in R$. In both cases $T=0$.

Proof. We will restrict our attention on the relation (1), the proof in case we have the relation (2) is similar and will therefore be omitted.

Linearization of the relation (1) gives

$$
T(x y z+z y x)=T(x y) z+T(z y) x-x y T(z)-z y T(x), x, y, z \in R
$$

and in particular for $z=x^{2}$

(3) $T\left(x y x^{2}+x^{2} y x\right)=T(x y) x^{2}+T\left(x^{2} y\right) x-x y T\left(x^{2}\right)-x^{2} y T(x), x, y \in R$.

Putting $x y+y x$ for $y$ in (1) and applying the relation (1) we obtain

$$
\begin{aligned}
T\left(x y x^{2}+x^{2} y x\right) & =T\left(x^{2} y+x y x\right) x-x^{2} y T(x)-x y x T(x) \\
= & T\left(x^{2} y\right) x+T(x y) x^{2}-x y T(x) x-x^{2} y T(x)-x y x T(x), x, y \in R .
\end{aligned}
$$

We have therefore

(4)

$T\left(x y x^{2}+x^{2} y x\right)=T\left(x^{2} y\right) x+T(x y) x^{2}-x y T(x) x-x^{2} y T(x)-x y x T(x), x, y \in R$.

By comparing (3) and (4) we obtain

$$
x y A(x)=0, x, y \in R,
$$

where $A(x)=T\left(x^{2}\right)-T(x) x-x T(x)$. Right multiplication of the above relation by $x$ and left multiplication by $A(x)$ gives $A(x) x y A(x) x=0$ for all pairs $x, y \in R$, whence it follows

$$
A(x) x=0, x \in R
$$

by semiprimeness of $R$. The substitution $A(x) y x$ for $y$ in the relation (5) gives $x A(x) y x A(x)=0$ for all pairs $x, y \in R$, which gives

$$
x A(x)=0, x \in R .
$$


The linearization of the relation (6) gives

$$
B(x, y) x+A(x) y+B(y, x) y+A(y) x=0
$$

for all pairs $x, y \in R$, where $B(x, y)$ denotes $T(x y+y x)-T(x) y-x T(y)-$ $T(y) x-y T(x)$. Putting in the above relation $-x$ for $x$ and comparing the relation so obtained with the above relation, one obtains

$$
B(x, y) x+A(x) y=0 .
$$

Right multiplication of the above relation by $A(x)$ gives, because of the relation (7), $A(x) y A(x)=0$ for all pairs $x, y \in R$, whence it follows $A(x)=0$ for all $x \in R$. In other words, $T$ is a Jordan derivation. By Cusack's generalization of Herstein's theorem $T$ is a derivation. From the relation (1) we obtain $T(x) y x+x T(y) x+x y T(x)=T(x) y x+x T(y) x-x y T(x)$, which reduces to

$$
x y T(x)=0, x, y \in R .
$$

From the relation (8) one obtains

$$
T(x) x=0, x \in R
$$

and

$$
x T(x)=0, x \in R,
$$

(see how the relations (6) and (7) were obtained from the relation (5)). The linearization of the relation (9) gives

$$
T(y) x+T(x) y=0, x, y \in R .
$$

Right multiplication of the above relation by $T(x)$ gives because of the relation (10) $T(x) y T(x)=0$ for all pairs $x, y \in R$, which gives $T=0$.

We proceed with the following corollary, which is a generalization of Theorem 4.

COROllary 6. Let $R$ be a 2 -torsion free semiprime ring and let $D ; G$ : $R \rightarrow R$ be additive mappings satisfying either the relations

$$
\begin{aligned}
& D(x y x)=D(x y) x+x y G(x), \\
& G(x y x)=G(x y) x+x y D(x)
\end{aligned}
$$

or the relations

$$
\begin{aligned}
& D(x y x)=D(x) y x+x G(y x), \\
& G(x y x)=G(x) y x+x D(y x)
\end{aligned}
$$

for all pairs $x, y \in R$. In both cases $D$ and $G$ are derivations and $D=G$. 
Proof. We will restrict our attention on the relations (11), the proof in case we have the relations (12) is similar and will therefore be omitted. We have therefore the relations

$$
\begin{aligned}
& D(x y x)=D(x y) x+x y G(x), x, y \in R, \\
& G(x y x)=G(x y) x+x y D(x), x, y \in R .
\end{aligned}
$$

Subtracting the relation (14) from the relation (13) one obtains

$$
T(x y x)=T(x y) x-x y T(x), x, y \in R,
$$

where $T(x)$ denotes $D(x)-G(x)$. Applying Theorem 5 gives $D=G$, which reduces the relation (13) to

$$
D(x y x)=D(x y) x+x y D(x), x, y \in R .
$$

Now, it follows from Theorem 4 that $D$ is a derivation, which completes the proof.

We proceed with the following conjecture.

CONJECTURe 7. Let $R$ be a semiprime ring with suitable torsion restrictions and let $D, G: R \rightarrow R$ be additive mappings satisfying either the relations

$$
\begin{aligned}
& D\left(x^{3}\right)=D\left(x^{2}\right) x+x^{2} G(x), \\
& G\left(x^{3}\right)=G\left(x^{2}\right) x+x^{2} D(x)
\end{aligned}
$$

or the relations

$$
\begin{aligned}
& D\left(x^{3}\right)=D(x) x^{2}+x G\left(x^{2}\right), \\
& G\left(x^{3}\right)=G(x) x^{2}+x D\left(x^{2}\right)
\end{aligned}
$$

for all $x \in R$. In both cases $D$ an $G$ are derivations and $D=G$.

Our next result is related to the conjecture above.

TheOREM 8. Let $X$ be a real or complex Banach space and let $A(X)$ be a standard operator algebra on $X$. Suppose there exist linear mappings $D, G: A(X) \rightarrow L(X)$ satisfying either the relations

$$
\begin{aligned}
& D\left(A^{3}\right)=D\left(A^{2}\right) A+A^{2} G(A), \\
& G\left(A^{3}\right)=G\left(A^{2}\right) A+A^{2} D(A)
\end{aligned}
$$

or the relations

$$
\begin{aligned}
& D\left(A^{3}\right)=D(A) A^{2}+A G\left(A^{2}\right), \\
& G\left(A^{3}\right)=G(A) A^{2}+A D\left(A^{2}\right)
\end{aligned}
$$

for all $A \in A(X)$. In both cases there exists some fixed $B \in L(X)$, such that $D(A)=G(A)=[A, B]$ holds for all $A \in A(X)$, which means that $D$ and $G$ are linear derivations. 
Theorem 8 is related to the result below first proved by Chernoff ([7]) (see also $[12-14,17-19])$.

Theorem 9. Let $X$ be a real or complex Banach space, let $A(X)$ be a standard operator algebra on $X$ and let $D: A(X) \rightarrow L(X)$ be a linear derivation. In this case $D$ is of the form $D(A)=[A, B]$ for all $A \in A(X)$ and some fixed $B \in L(X)$.

In the proof of Theorem 8 we use Herstein's theorem, Theorem 9, Lemma 10 and methods which are similar to those used in [17 - 20].

LEMMA 10. Let $R$ be a semiprime ring and let $f: R \rightarrow R$ be an additive mapping. If either

$$
f(x) x=0
$$

or

$$
x f(x)=0
$$

holds for all $x \in R$, then $f=0$.

For the proof of Lemma 10 we refer to [15].

Proof of Theorem 8. We will focus our attention to the relations (16). The proof in case we have the relations (17) is similar and will therefore be omitted. We have therefore the relations

$$
\begin{aligned}
& D\left(A^{3}\right)=D\left(A^{2}\right) A+A^{2} G(A), \\
& G\left(A^{3}\right)=G\left(A^{2}\right) A+A^{2} D(A)
\end{aligned}
$$

for all $A \in A(X)$. Subtracting the relation (19) from the relation (18), we obtain

$$
T\left(A^{3}\right)=T\left(A^{2}\right) A-A^{2} T(A)
$$

for all $A \in A(X)$, where $T(A)$ denotes $D(A)-G(A)$. It is our aim to show that $T(A)=0$ for all $A \in A(X)$. Let us first consider the restriction of $T$ on $F(X)$. Let $A$ be from $F(X)$ and $P \in F(X)$ a projection with $A P=P A=A$. Putting $P$ for $A$ in relation (20), we obtain

$$
T(P)=T(P) P-P T(P) .
$$

Right multiplication of the relation $(21)$ by $P$ gives

$$
P T(P) P=0 \text {. }
$$

Left multiplication of the relation $(21)$ by $P$ gives because of $(22)$

$$
P T(P)=0 \text {. }
$$

Putting $A+P$ for $A$ in the relation (20), we obtain after some calculations

$$
\begin{aligned}
3 T\left(A^{2}\right)+3 T(A)= & T\left(A^{2}\right) P+2 T(A) A+2 T(A) P+T(P) A \\
& -A^{2} T(P)-2 A T(A)-2 A T(P)-P T(A) .
\end{aligned}
$$


Putting $-A$ for $A$ in the above relation and comparing the relation so obtained with the above relation, we obtain

$$
3 T(A)=2 T(A) P+T(P) A-2 A T(P)-P T(A) .
$$

Left multiplication of the relation (23) by $A$ and considering $A=A P$ gives $A T(P)=A P T(P)=0$, which reduces the relation above to

$$
3 T(A)=2 T(A) P+T(P) A-P T(A) .
$$

Multiplying the relation (24) from both sides by $P$ and considering (23), we obtain

$$
P T(A) P=0 .
$$

Left multiplication of the relation (24) by $P$ and considering both (23) and (25) leads us to

$$
P T(A)=0 .
$$

Left multiplication of the relation (26) by $A$ gives

$$
A T(A)=0 .
$$

Applying (26) in the relation (24) leads us to

$$
3 T(A)=2 T(A) P+T(P) A .
$$

Right multiplication of the relation (28) by $P$ gives $T(A) P=T(P) A$, which reduces the relation (28) to

$$
T(A)=T(A) P .
$$

From the relation (29) one can conclude that $T$ maps $F(X)$ into itself. We have therefore a linear mapping $T$, which maps $F(X)$ into itself, satisfying the relation (27). Applying Lemma 10 we can conclude that $T(A)=0$ for all $A \in F(X)$. It remains to prove that $T(A)=0$ holds for all $A \in A(X)$ as well. The mapping $T$ on $A(X)$ is linear, satisfies the relation (20) and, as we already showed, vanishes on $F(X)$. Let $A \in A(X)$, let $P$ be a one-dimensional projection and let us introduce $S \in A(X)$ by $S=A+P A P-(A P+P A)$. We have $S P=P S=0$. It is easy to see that $T(S)=T(A)$ and $T\left(S^{2}\right)=T\left(A^{2}\right)$. By the equation (20) we now have

$$
\begin{aligned}
& T\left(S^{2}\right) S-S^{2} T(S)=T\left(S^{3}\right)=T\left(S^{3}+P\right) \\
& \quad=T\left((S+P)^{3}\right)=T\left(\left(S^{2}\right)(S+P)-(S+P)^{2} T(S)\right. \\
& \quad=T\left(S^{2}\right) S+T\left(S^{2}\right) P-S^{2} T(S)-P T(S) .
\end{aligned}
$$

We have therefore $T\left(S^{2}\right) P-P T(S)=0$ and since $T(S)=T(A)$ and $T\left(S^{2}\right)=$ $T\left(A^{2}\right)$, we obtain

$$
T\left(A^{2}\right) P-P T(A)=0 .
$$


Putting $-A$ for $A$ in the relation (30) and comparing the relation so obtained with (30), we get

$$
P T(A)=0 .
$$

As $P$ is an arbitrary one-dimensional projection, we have $T(A)=0$ for all $A \in A(X)$, which was our intention to prove. We have therefore proved that $D=G$. This ascertainment enables us to combine (18) and (19) into one relation

$$
D\left(A^{3}\right)=D\left(A^{2}\right) A+A^{2} D(A) .
$$

Let us first consider the restriction of $D$ on $F(X)$. Let $A$ be from $F(X)$ and let $P \in F(X)$ be a projection with $A P=P A=A$. Putting $P$ for $A$ in the relation (32), we obtain $D(P)=D(P) P+P D(P)$. Any multiplication of the last relation by $P$ leads us to

$$
P D(P) P=0 .
$$

The substitution $A+P$ for $A$ in the relation (32) gives

$$
\begin{aligned}
3 D\left(A^{2}\right)+3 D(A)+D(P) & \\
= & D\left(A^{2}\right) P+2 D(A) P+2 D(A) A+D(P) A+D(P) P \\
& +A^{2} D(P)+2 A D(A)+2 A D(P)+P D(A)+P D(P) .
\end{aligned}
$$

Previously mentioned relation $D(P)=D(P) P+P D(P)$ reduces the equation (34) to

$$
\begin{aligned}
3 D\left(A^{2}\right)+3 D(A)= & D\left(A^{2}\right) P+2 D(A) P+2 D(A) A+D(P) A \\
& +A^{2} D(P)+2 A D(A)+2 A D(P)+P D(A) .
\end{aligned}
$$

Putting $-A$ for $A$ in the above relation and comparing the relation so obtained with the above relation, we obtain

$$
3 D\left(A^{2}\right)=D\left(A^{2}\right) P+2 D(A) A+A^{2} D(P)+2 A D(A)
$$

and

$$
3 D(A)=2 D(A) P+D(P) A+2 A D(P)+P D(A) .
$$

Right multiplication of the relation (35) by $P$ gives

$$
3 D\left(A^{2}\right) P=D\left(A^{2}\right) P+2 D(A) A+A^{2} D(P) P+2 A D(A) P,
$$

which is, after considering (33) and dividing by 2 , reduced to

$$
D\left(A^{2}\right) P=D(A) A+A D(A) P .
$$

The relation (35) gives because of (38)

$$
3 D\left(A^{2}\right)=3 D(A) A+A D(A) P+A^{2} D(P)+2 A D(A) .
$$


Left multiplication of the relation (36) by $A$, considering (33) and dividing by 2 leads us to

$$
A D(A)=A D(A) P+A^{2} D(P) .
$$

The relation (39) gives because of (40)

$$
3 D\left(A^{2}\right)=3 D(A) A+3 A D(A),
$$

which reduces to

$$
D\left(A^{2}\right)=D(A) A+A D(A) .
$$

From the relation (36) one can conclude that $D$ is a linear mapping, which maps $F(X)$ into itself. By the relation (41) $D$ is a Jordan derivation on $F(X)$. Since $F(X)$ is prime, it follows that $D$ is a derivation by Herstein's theorem. Applying Theorem 9 one can conclude that $D$ is of the form

$$
D(A)=[A, B]
$$

for all $A \in F(X)$ and some fixed $B \in L(X)$. It remains to prove that the relation (42) holds for all $A \in A(X)$ as well. For this purpose we introduce $D_{1}: A(X) \longrightarrow L(X)$ by $D_{1}(A)=[A, B]$ and consider $D_{0}=D-D_{1}$. The mapping $D_{0}$ is linear, satisfies the relation (32) and it vanishes on $F(X)$. Our aim is to prove that $D_{0}$ vanishes on $A(X)$ as well. Let $A \in A(X)$, let $P$ be a one-dimensional projection and let us introduce $S \in A(X)$ by $S=A+P A P-(A P+P A)$. We have $S P=P S=0$. It is easy to see that $D_{0}(S)=D_{0}(A)$ and $D_{0}\left(S^{2}\right)=D_{0}\left(A^{2}\right)$. The relation (32) now leads us to

$$
\begin{aligned}
D_{0}\left(S^{2}\right) S+S^{2} D_{0}(S) & =D_{0}\left(S^{3}\right)=D_{0}\left(S^{3}+P\right)=D_{0}\left((S+P)^{3}\right) \\
& =D_{0}\left(S^{2}\right) S+D_{0}\left(S^{2}\right) P+S^{2} D_{0}(S)+P D_{0}(S) .
\end{aligned}
$$

We have therefore $D_{0}\left(S^{2}\right) P+P D_{0}(S)=0$ and since $D_{0}(S)=D_{0}(A)$ and $D_{0}\left(S^{2}\right)=D_{0}\left(A^{2}\right)$, we arrive at

$$
D_{0}\left(A^{2}\right) P+P D_{0}(A)=0 .
$$

Putting $-A$ for $A$ in the relation (43) and subtracting the relation so obtained from the relation (43), we get

$$
P D_{0}(A)=0 .
$$

Since $P$ is an arbitrary one-dimensional projection, it follows that $D_{0}(A)=0$ for all $A \in A(X)$, which concludes the proof of the theorem.

\section{ACKNOWLEDGEMENTS.}

The author wishes to express his thanks to Professor Joso Vukman for proposing the problem and for helpful discussion. 


\section{REFERENCES}

[1] K. I. Beidar, M. Brešar, M. A. Chebotar and W. S. Martindale III, On Herstein's Lie map Conjectures. II, J. Algebra 238 (2001), 239-264.

[2] K. I. Beidar, W. S. Martindale III and A. V. Mikhalev, Rings with generalized identities, Marcel Dekker, Inc., New York, 1996.

[3] M. Brešar, Jordan derivations on semiprime rings, Proc. Amer. Math. Soc. 104 (1988), 1003-1006.

[4] M. Brešar, Jordan mappings of semiprime rings, J. Algebra 127 (1989), 218-228.

[5] M. Brešar and J. Vukman, Jordan derivations on prime rings, Bull. Austral. Math. Soc. Vol. 37 (1988), 321-322.

[6] M. Brešar and J. Vukman, Jordan $(\theta, \phi)$-derivations, Glas. Mat. Ser. III 28(48) (1991), 83-88.

[7] P. R. Chernoff, Representations, automorphisms and derivations of some Operator Algebras, J. Functional Analysis 12 (1973), 275-289.

[8] J. Cusack, Jordan derivations on rings, Proc. Amer. Math. Soc. 53 (1975), 321-324.

[9] I. N. Herstein, Jordan derivations on prime rings, Proc. Amer. Math. Soc. 8 (1957), 1104-1119.

[10] I. Kosi-Ulbl and J. Vukman, A note on derivations in semiprime rings, Int. J. Math. Math. Sci. 20 (2005), 3347-3350.

[11] I. Kosi-Ulbl and J. Vukman, On derivations in rings with involution, Int. Math. J. 6 (2005), 81-91.

[12] I. Kosi-Ulbl and J. Vukman, An identity related to derivations of standard operator algebras and semisimple $H^{*}$-algebras, Cubo 12 (2010), 95-102.

[13] P. Šemrl, Ring derivations on standard operator algebras, J. Functional Analysis 112 (1993), 318-324.

[14] J. Vukman, On automorphisms and derivations of operator algebras, Glas. Mat. Ser. III 19(39) (1984), 135-138.

[15] J. Vukman, Identities with derivations and automorphisms on semiprime rings, Int. J. Math. Math. Sci. (2005), 1031-1038.

[16] J. Vukman, I. Kosi-Ulbl and D. Eremita, On certain equations in rings, Bull. Austral. Math. Soc. Vol. 71 (2005), 53-60.

[17] J. Vukman, On derivations of algebras with involution, Acta Math. Hungar. 112 (2006), 181-186.

[18] J. Vukman, On derivations of standard operator algebras and semisimple $H^{*}$ algebras, Studia Sci. Math. Hungar. 44 (2007), 57-63.

[19] J. Vukman, Identities related to derivations and centralizers on standard operator algebras, Taiwanese J. Math. 11 (2007), 255-265.

[20] J. Vukman, Some remarks on derivations in semiprime rings and standard operator algebras, Glas. Mat. Ser. III 46(66) (2011), 43-48.

N. Širovnik

Department of Mathematics and Computer Science

Faculty of Natural Sciences and Mathematics

University of Maribor

Koroška 160, 2000 Maribor

Slovenia

E-mail: nejc.sirovnik@uni-mb.si

Received: 14.10 .2010

Revised: 22.2.2011. 